\title{
Commentary Standardisation of data collection in traumatic brain injury: key to the future?
}

\author{
Andrew IR Maas
}

Department of Neurosurgery, University Hospital Antwerp, Wilrijkstraat 10, 2650 Edegem, Belgium

Corresponding author: Andrew IR Maas, andrew.maas@uza.be

Published: 16 December 2009

This article is online at http://ccforum.com/content/13/6/1016

(c) 2009 BioMed Central Ltd
Critical Care 2009, 13:1016 (doi:10.1186/cc8163)

\begin{abstract}
Great variability exists in data collection and coding of variables in studies on traumatic brain injury (TBI). This confounds comparison of results and analysis of data across studies. The difficulties in performing a meta-analysis of individual patient data were recently illustrated in the IMPACT project (International Mission on Prognosis and Clinical Trial Design in TBI): merging data from 11 studies involved over 10 person years of work. However, these studies did confirm the great potential for advancing the field by this approach. Although randomized controlled trials remain the prime approach for investigating treatment effects, these can never address the many uncertainties concerning multiple treatment modalities in TBI. Pooling data from different studies may provide the best possible source of evidence we can get in a cost efficient way. Standardisation of data collection and coding is essential to this purpose. Recommendations hereto have been proposed by an interagency initiative in the US. These proposals deserve to be taken forward at an international level. This initiative may well constitute one of the most important steps forwards, paving the road for harvesting successful results in the near future.
\end{abstract}

Traumatic brain injury (TBI) is a field in medicine with one of the greatest unmet needs [1]. Severe injuries constitute a leading cause of death and disability worldwide, with devastating effects on patients and their relatives and high socioeconomic costs. TBI is a heterogeneous disease in terms of cause, pathology, severity and prognosis. Procedures for data collection and coding of variables in TBI studies are equally heterogeneous. This was recently illustrated in the IMPACT project (International Mission on Prognosis and Clinical Trial Design in TBI) in which individual patient data from three observational series and eight clinical trials were merged into a large registry, forming a culture medium for exploring concepts to improve the design of clinical trials in TBI [2]. Creating this registry involved over 10 person years of work due to the widely differing structure of the study datasets, poor documentation and variability in coding. Lack of standardization has been a major factor confounding comparisons between studies, and complicating metaanalyses of individual patient data.

Analysing individual patient data across studies may well be key to advancing the clinical field of TBI, and improving treatment. Much uncertainty exists regarding the benefit and risk of many treatment modalities in TBI. This uncertainty is reflected in the paucity of class I and II evidence underpinning authoritative guideline recommendations [3]. Although randomized controlled trials remain the prime approach for investigating treatment effects, these are costly and logistically demanding. Consequently, it seems unlikely that we can mount adequately powered trials to study all relevant treatment modalities. Pooling data from multiple studies can provide an alternative source of evidence that can be realistically obtained in a cost-efficient way. Relating differences in trauma organization and treatment approaches to outcome will permit both better targeting of prevention and exploration of reasons for observed differences. Further, this approach provides a means of generating and refining hypotheses, and ranking them in importance for testing.

The great potential of performing a meta-analysis of individual patient data was demonstrated by the IMPACT studies. Simulation studies showed that the statistical power in TBI trials may be increased up to $50 \%$ by utilizing more efficient approaches to the analysis [4]. Extensive prognostic analysis defined the strength of many known predictors more precisely, yielded new predictors and has resulted in validated prognostic models for use in moderate and severe TBI [5]. The benefit of analyzing large numbers of patients was also demonstrated in the development of prognostic models based on the CRASH trial [6]. These models are useful for providing information on expectations of outcome, for classifying the severity of brain injury, for stratification and covariate adjustment in clinical trials, and as reference for evaluating quality of care.

$\mathrm{TBI}=$ traumatic brain injury. 
Standardization of data collection and coding is essential to facilitate sharing of results and to analyze data across studies.

Initial steps undertaken by the IMPACT study group towards development of standardization were integrated in the US into a much larger interagency initiative towards 'an integrated approach to research in psychological health and traumatic brain injury'. This initiative included working groups on demographics and clinical assessment, biomarkers, neuroimaging and outcome. The global aim was to develop recommendations on selection and coding of data elements for studies across the broad spectrum of TBI.

The process was consensus driven, with multidisciplinary input from a broad range of experts, covering the entire trauma chain from emergency medicine to rehabilitation and late outpatient care. Recommendations were formulated and templates produced summarizing coding formats, motivation of choices and procedures. The data elements are contained in modules, which are grouped in categories. For example, the data elements 'age, gender and race' are contained in the module 'demographics' under the category 'subject characteristics'. As the required level of detail may vary greatly with the aim of a specific study, three versions for coding data elements were developed: a basic, an advanced, and an extended format with the greatest level of detail in the extended version. The coding of these versions is such that more detailed coding can be collapsed into the basic version, thus facilitating comparison across studies. The draft recommendations and templates are available from the author and will be posted on the web in early 2010 [7].

This work presents a major advance towards standardisation, but has not yet addressed approaches to analysis of parameters such as intracranial pressure that are continuously monitored in the ICU setting. Here, approaches are often crude and widely diverging, using only momentary or summary measures. Few studies have taken advantage of the more extensive information contained in continuous monitoring. We strongly advocate further development of software aimed at capturing the frequency distribution of measured values during continuous monitoring and further research into the best approaches to analysis.

These developments may well constitute one of the most important steps forward in the field of clinical trials in TBI, paving the road for harvesting successful results in the near future.

\section{Competing interests}

Grant support was provided by NIH-NINDS (NS 042691) and further funded as part of the interagency initiative in the US towards 'an integrated approach to research in Psychological Health and Traumatic Brain Injury'.

\section{Acknowledgements}

This commentary is based upon extensive collaborative work, performed by the IMPACT study group and the interagency working group on Demographics and Clinical Assessment. Grant support was provided by NIH-NINDS (NS 042691) and further funded as part of the interagency initiative in the US towards 'an integrated approach to research in Psychological Health and Traumatic Brain Injury'.

\section{References}

1. Maas AIR, Stocchetti N, Bullock R: Moderate and severe traumatic brain injury in adults. Lancet Neuro/ 2008, 7:728-741.

2. Maas Al, Marmarou A, Murray GD, Teasdale SG, Steyerberg EW: Prognosis and clinical trial design in traumatic brain injury: the IMPACT study. J Neurotrauma 2007, 24:232-238.

3. Brain Trauma Foundation [http://www.braintrauma.org/site/ PageServer]

4. Maas Al, Steyerberg EW, Marmarou A, McHugh GS, Lingsma HF, Butcher I, Lu J, Weir J, Roozenbeek B, Murray GD: IMPACT recommendations for improving the design and analysis of clinical trials in moderate to severe traumatic brain injury. Neurotherapeutics, in press.

5. Steyerberg EW, Mushkudiani N, Perel P, Butcher I, Lu J, McHugh GS, Murray GD, Marmarou A, Roberts I, Habbema JD, Maas Al: Predicting outcome after traumatic brain injury: development and international validation of prognostic scores based on admission characteristics. PLoS Med 2008, 5:e165.

6. MRC CRASH Trial Collaborators, Perel P, Arango M, Clayton T, Edwards P, Komolafe E, Poccock S, Roberts I, Shakur H, Steyerberg E, Yutthakasemsunt S: Predicting outcome after traumatic brain injury: practical prognostic models based on large cohort of international patients. BMJ 2008, 336:425-429.

7. [www.nindscommondataelements.org] 